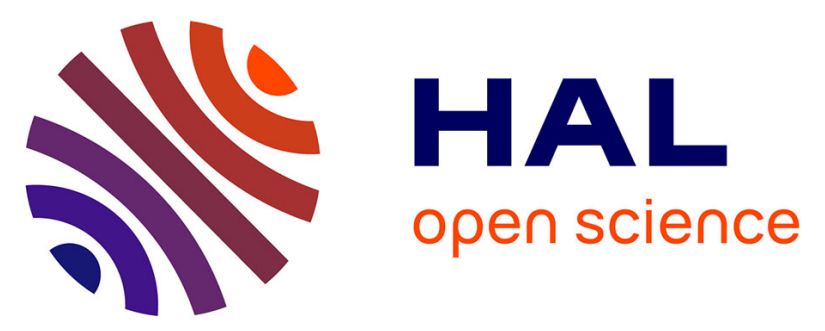

\title{
Gene expression profiling of leukemia T-cells resistant to methotrexate and 7 -hydroxymethotrexate reveals alterations that preserve intracellular levels of folate and nucleotide biosynthesis
}

\author{
Alan Kambiz Fotoohi, Yehuda G. Assaraf, Ali Moshfegh, Jamileh Hashemi, \\ Gerrit Jansen, Godefridus J. Peters, Catharina Larsson, Freidoun Albertioni
}

\section{To cite this version:}

Alan Kambiz Fotoohi, Yehuda G. Assaraf, Ali Moshfegh, Jamileh Hashemi, Gerrit Jansen, et al.. Gene expression profiling of leukemia T-cells resistant to methotrexate and 7-hydroxymethotrexate reveals alterations that preserve intracellular levels of folate and nucleotide biosynthesis. Biochemical Pharmacology, 2009, 77 (8), pp.1410. 10.1016/j.bcp.2008.12.026 . hal-00493484

\author{
HAL Id: hal-00493484 \\ https://hal.science/hal-00493484
}

Submitted on 19 Jun 2010

HAL is a multi-disciplinary open access archive for the deposit and dissemination of scientific research documents, whether they are published or not. The documents may come from teaching and research institutions in France or abroad, or from public or private research centers.
L'archive ouverte pluridisciplinaire HAL, est destinée au dépôt et à la diffusion de documents scientifiques de niveau recherche, publiés ou non, émanant des établissements d'enseignement et de recherche français ou étrangers, des laboratoires publics ou privés. 


\section{Accepted Manuscript}

Title: Gene expression profiling of leukemia T-cells resistant to methotrexate and 7-hydroxymethotrexate reveals alterations that preserve intracellular levels of folate and nucleotide biosynthesis

Authors: Alan Kambiz Fotoohi, Yehuda G. Assaraf, Ali Moshfegh, Jamileh Hashemi, Gerrit Jansen, Godefridus J. Peters, Catharina Larsson, Freidoun Albertioni<ce:footnote id="fn1" $><$ ce:note-para $>$ These authors have contributed equally to the paper. $</$ ce:note-para $></$ ce:footnote $>$

PII: S0006-2952(09)00004-5

DOI: doi:10.1016/j.bcp.2008.12.026

Reference: $\quad$ BCP 10059

To appear in: $\quad B C P$

Received date: $\quad 20-11-2008$

Revised date: $\quad 30-12-2008$

Accepted date: $\quad 30-12-2008$

Please cite this article as: Fotoohi AK, Assaraf YG, Moshfegh A, Hashemi J, Jansen G, Peters GJ, Larsson C, Albertioni F, Gene expression profiling of leukemia T-cells resistant to methotrexate and 7-hydroxymethotrexate reveals alterations that preserve intracellular levels of folate and nucleotide biosynthesis, Biochemical Pharmacology (2008), doi:10.1016/j.bcp.2008.12.026

This is a PDF file of an unedited manuscript that has been accepted for publication. As a service to our customers we are providing this early version of the manuscript. The manuscript will undergo copyediting, typesetting, and review of the resulting proof before it is published in its final form. Please note that during the production process errors may be discovered which could affect the content, and all legal disclaimers that apply to the journal pertain. 


\section{Gene expression profiling of leukemia T-cells resistant to methotrexate and}

\section{7-hydroxymethotrexate reveals alterations that preserve intracellular levels of}

\section{folate and nucleotide biosynthesis}

Alan Kambiz Fotoohi, ${ }^{1, *}$ Yehuda G. Assaraf, ${ }^{2, *}$ Ali Moshfegh, ${ }^{1, \#}$ Jamileh Hashemi, ${ }^{3, \#}$ Gerrit Jansen, ${ }^{4}$ Godefridus J. Peters, ${ }^{5}$ Catharina Larsson, ${ }^{3}$ and Freidoun Albertioni ${ }^{1}$

${ }^{1}$ Department of Oncology and Pathology, Karolinska Institutet, Cancer Center Karolinska, Karolinska University Hospital, Stockholm, Sweden;

${ }^{2}$ The Fred Wyszkowski Cancer Research Laboratory, Department of Biology, TechnionIsrael Institute of Technology, Haifa, Israel;

${ }^{3}$ Department of Molecular Medicine and Surgery, Karolinska Institutet, CMM Karolinska University Hospital, Stockholm, Sweden;

${ }^{4}$ Department of Rheumatology, University Hospital Vrije Universteit, Amsterdam, the Netherlands;

${ }^{5}$ Department of Medical Oncology, VU University Medical Center, Amsterdam, the Netherlands

*, These authors have contributed equally to the paper

Key words: Methotrexate, 7-hydroxymethotrexate, resistance, leukemia, folylpolyglutamate synthetase, reduced folate carrier, thymidylate synthase, microarray, real-time quantitative RT-PCR

Running title: Expression profiles of 7-OHMTX and MTX-resistant cells

Abbreviations: MTX, methotrexate; 7-OHMTX, 7-hydroxymethotrexate; $\mathrm{IC}_{50}$, the drug concentration inhibiting cell growth by 50\%; DHFR, dihydrofolate reductase; TS, thymidylate synthase; RFC, reduced folate carrier; FPGS, folylpolyglutamate synthetase; FPGH, folylpolyglutamate hydrolase; GARFT, glycinamide ribonucleotide formyltransferase, MRP, multidrug resistance-associated protein.

Grant support: The Children Cancer Foundation, The Cancer and Allergy Foundation, The Cancer Society in Stockholm, The King Gustaf V Jubilee Fund, Swedish Medical Society, Swedish Cancer Foundation, Swedish Research Council, Göran Gustafsson Foundation for Research in Natural Sciences and Medicine, and Karolinska Institutet Foundations

Correspondence and requests for reprints to Freidoun Albertioni, Cancer Center Karolinska, Department of Oncology and Pathology, Karolinska Institutet, Karolinska University Hospital, Stockholm, Sweden, SE-171 76 Stockholm, Sweden.

Phone: +46 (0) 8517 75832; fax: +46 (0) 8517 75042; e-mail: freidoun.albertioni@ki.se 


\begin{abstract}
In vitro treatment of human T-cell leukemia cells with 7-hydroxymethotrexate, the major metabolite of methotrexate resulted in acquired resistance as a result of the complete loss of folypolyglutamate synthetase (FPGS) activity. This was in contradistinction to the major modality of antifolate resistance of impaired drug transport in leukemia cells exposed to methotrexate.. To identify the genes associated with methotrexate and 7hydroxymethotrexate resistance, we herein explored the patterns of genome-wide expression profiles in these antifolte-resistant leukemia sublines. mRNA levels of the reduced folate carrier, the primary influx transporter of folates and antifolates, were down-regulated $>2$-fold in methotrexate -resistant cells. The dramatic loss of FPGS activity in 7-hydroxymethotrexate - resistant cells was associated with alterations in the expression of various genes aimed at preserving reduced folates and/or enhancing purine nucleotide biosynthesis e.g. methylene tetrahydrofolate reductase, glycinamide ribonucleotide formyltransferase, adenosine deaminase, cystathionine $\beta$ synthase, as well as the ATP-dependent folate exporters $B C R P / A B C G 2$ and $M R P 1 / A B C C 1$. The observed changes in gene expression were generally not paralleled by acquired DNA copy numbers alterations, suggesting transcriptional regulatory mechanisms. Interestingly, gene expression of DNA/RNA metabolism and transport genes were more profoundly altered in methotrexate -resistant subline, whereas in 7-hydroxymethotrexate -resistant cells, the most profoundly affected groups of genes were those encoding for proteins involved in metabolism and cellular proliferation. Thus, the present investigation provides evidence that 7-hydroxymethotrexate induces gene expression alterations and an antifolate resistance modality that are distinct from its parent drug methotrexate.
\end{abstract}




\section{Introduction}

During the past six decades, the rate of success in curing childhood acute lymphoblastic leukemia (ALL) has increased from less than $10 \%$ to more than $80 \%$. This dramatic improvement is largely due to optimization of the use of existing chemotherapeutic agents. Methotrexate (MTX) is the major anti-metabolite (Fig. 1) employed in combination chemotherapeutic regimens for childhood ALL. Treatment failure is mainly related to acquired drug resistance or selection of pre-existing molecular alterations facilitating antifolate resistance.

The mechanisms of action and resistance to MTX and its major metabolite 7hydroxymethotrexate (7-OHMTX) have been characterized in some detail [1, 2]. Decreased cellular uptake via the reduced folate carrier (RFC/SLC19A1) [3] or increased active efflux mediated by $\mathrm{ABC}$ transporters such as the multidrug resistance-associated proteins 1-5 (MRP 1-5), the breast cancer resistance protein (BCRP/ABCG2) $[4,5]$ and P-glycoprotein (MDR or ABCB1) [6] can lead to resistance to hydrophilic and lipophilic antifolates. The ability of cells to accumulate long chain MTX polyglutamates (MTX-PGs) is highly dependent on the activities of folylpolyglutamate synthetase (FPGS) and folylpolyglutamate hydrolase (FPGH). Furthermore, the susceptibility of cells to the cytotoxic effect elicited by MTX may also be influenced by mutations in the dihydrofolate reductase gene $(D H F R)$ that result in a lower affinity for MTX, by increased activity of thymidylate synthase (TS), as well as by altered activities of other folate- and one-carbon metabolism enzymes. 
The metabolite 7-OHMTX (Fig. 1) results from oxidation of MTX primarily by hepatic aldehyde oxidase. Intracellular conversion of MTX to 7-OHMTX has also been demonstrated in leukemia cells [7] . Although the affinity of DHFR for 7-OHMTX is markedly lower (>100-fold) than for MTX [8], the polyglutamated forms of this metabolite are more potent inhibitors of this enzyme $[9,10]$. Polyglutamated forms of MTX and polyglutamated conjugates of 7-OHMTX are also capable of inhibiting a number of other folate-dependent enzymes involved in de novo purine biosynthesis such as TS, glycinamide ribonucleotide formyltransferase (GARFT) and aminoimidazole carboxamide ribonucleotide formyltransferase (AICART).

We have recently reported that prolonged exposure of human leukemic T-cells to 7-OHMTX leads to antifolate resistance that attenuates the efficacy of MTX [11]. While the primary mechanism of resistance to MTX was a marked reduction in RFC-mediated drug uptake, resistance to 7-OHMTX was due to a dramatic decrease (>98\%) in FPGS activity, which enhanced resistance to short-term (4 hr) exposure to MTX more than 100-fold. To further characterize the molecular mechanisms associated with acquired resistance to MTX and 7OHMTX in human leukemia cells, we characterized gene expression profiles and alterations in gene copy numbers using high density microarrays. The results revealed disparate cellular responses to MTX and 7-OHMTX that may facilitate the identification of novel cellular targets and modalities of resistance to 7-OHMTX as well as possibly suggesting novel approaches to overcome antifolate resistance and design individualized antifolate-based drug therapy of human leukemia. 


\section{Materials and Methods}

\subsection{Materials}

The HG-U133A GeneChip oligonucleotide microarray and all reagents required for microarray analysis were purchased from Affymetrix (Affymetrix Inc., Santa Clara, CA, USA). TaqMan reagents and gene expression assays were obtained from Applied Biosystems (Stockholm, Sweden) including: folylpolyglutamate synthetase (FPGS, No: Hs00191956 m1), folylpolyglutamate hydrolase ( $F P G H / G G H$, No: Hs00608257 m1), the reduced folate carrier $(R F C / S L C 19 A 1$, No: Hs00161870 m1), glycinamide ribonucleotide transformylase (GART, No: Hs00531926 m1), 5-aminoimidazole-4carboxamide ribonucleotide formyltransferase (AICART, No: Hs00269671 m1), 5methyltetrahydrofolate-homocysteine methyltransferase (MTR, No: Hs00165188_m1), 5,10-methylenetetrahydrofolate reductase (MTHFR, No: Hs00195560_m1), multidrug resistance-associated proteins 4 (MRP4, No: Hs00195260), and 5 (MRP5, No: Hs00194701_m1), and glyceraldehyde-3-phosphate dehydrogenase (GAPDH, No: Hs99999905_m1).

\subsection{Comparison of plasma MTX and 7-OHMTX levels in MTX treated ALL patients}

Plasma levels of MTX and 7-OHMTX previously determined in 49 childhood ALL patients by HPLC [12] . The conditions for sample collections and procedures and HPLC assay applied are according to previously published information [13]. In short, plasma samples were obtained after intravenous infusion of MTX $\left(5-8 \mathrm{~g} / \mathrm{m}^{2}\right)$ during 24 hours. Blood samples were collected 20-23 h after beginning of MTX infusion (steady-state concentration), at $36 \mathrm{~h}$ and once every 6 hours afterward until plasma-MTX concentration fell below $<0.2 \mu \mathrm{M}$. Samples were generally drawn from a central venous catheter, while the steady-state samples were obtained from a peripheral vein.

\subsection{Antifolate-resistant cells and tissue culturing}

MOLT-4 cells derived from a human T-cell ALL were purchased from American Type Culture Collection (Rockville, MD, USA). The generation and functional characterization of drug resistant derivatives have been previously reported [11]. In short, this involved sequential exposure (12-18 steps each of 4 days) of parental MOLT-4 cells to gradually increasing concentrations of MTX (1-300 nM) or 7-OHMTX (50-30,000 nM). This resulted in sub-lines with greater than 50-fold increased resistance to MTX or 7-OHMTX relative to parental cells. Cells were cultured in RPMI-1640 medium (supplemented with 10\% fetal calf serum, $100 \mathrm{U} / \mathrm{ml}$ penicillin, $100 \mu \mathrm{g} / \mathrm{ml}$ streptomycin, and $2 \mathrm{mM} \mathrm{L}$-glutamine) at $37{ }^{\circ} \mathrm{C}$ under 
a humidified air containing 5\% $\mathrm{CO}_{2}$. For experimental studies cells were cultured in drugfree-medium for at least 3 passages, and harvested during the logarithmic phase growth at a density $0.8-1.5 \times 10^{6} / \mathrm{ml}$ as determined by cell counting using a Coulter Multi-sizer (Coulter Electronics, Luton, United Kingdom).

\subsection{Isolation and Quality Verification of RNA}

Triplicate inoculates of parental and resistant cells were thawed, washed and cultured in a standard medium (see above) for 4 passages, following which $10^{7}$ cells of each cell type were collected by centrifugation. High quality RNA was extracted using the RNeasy Midi kit in accordance with the manufacturer's instructions (RNeasy Midi Handbook; Qiagen, KEBO Lab, Spånga, Sweden). A NanoDrop ND-1000 UV-Vis Spectrophotometer (NanoDrop Technologies Wilmington, DE) was used to determine RNA concentrations, and to verify its quality and purity, whereby RNA with an OD ratio of 1.99-2.0 at 260/280 was found acceptable.

\subsection{Experimental Conditions and Analysis of Expression Arrays}

The experimental procedures and analyses were in accordance with the instructions of the manufacturer (Technical manual of Affymetrix GeneChip products). In short, complementary DNA (cDNA) synthesized from $11 \mu \mathrm{g}$ of total RNA, was used for synthesis and isolation of biotin-labelled complementary RNA (cRNA), and fragmented to a mean size of $\sim 50-100$ nucleotides. Samples were subsequently analyzed in triplicate on Affymetrix U133A GeneChips containing 22,283 sets of probes for approximately 17,000 different species of mRNA. After hybridization for $16 \mathrm{~h}$ at $45^{\circ} \mathrm{C}$, washing and staining with streptavidin-R-phycoerythrin, the arrays were scanned in an Agilent Gene Array Scanner 
(Affymetrix). The arrays were first analyzed using GeneChip Operating Software (GCOS 1.4; Affymetrix), and data files were subsequently analyzed utilizing the GeneSpring 7.2 software. Normalization was performed with the same software employing default normalization parameters as follows: for each sample the raw data were divided by the 50 th percentile of all measurements, and for each gene the raw data were divided by the median level of expression of the specific samples in question. Data from sets of probes designed to detect the expression of genes that failed to fulfil the criteria for detection (labelled "absent" or "marginal" in all microarrays) and an intensity value less than 50 (to reduce background signals) were eliminated. For statistical calculations, analysis of variance (ANOVA) were used $(\mathrm{p}<0.05)$, and Benjamin and Hochberg false discovery rate were used for multiple testing correction. After the filtration steps, 863 probe sets with significantly altered expression levels between parental and/or MTX resistant and/or 7-OHMTX resistant cells remained for further analysis (Fig. 3). All microarray data were submitted to http://www.ncbi.nlm.nih.gov/geo/.

\subsection{Quantitative Real-time PCR (qRT-PCR)}

qRT-PCR was performed on cDNA samples of parental and resistant cells in three independent experiments to quantify the expression of $F P G S, F P G H / G G H, R F C / S L C 19 A 1, G A R T, A I C A R T, M T R, M T H F R, M R P 4$, and MRP5. GAPDH was analysed as a positive control as well as used for normalization.

qRT-PCR reactions were carried out in $20 \mu \mathrm{l}$ mixtures containing $9 \mu \mathrm{l}$ cDNA template, $1 \mu \mathrm{l}$ TaqMan ${ }^{\circledR}$ Gene Expression Assay and $10 \mu \mathrm{l}$ TaqMan Fast Universal PCR Master Mix, No AmpErase UNG concentrated 2-fold. Amplifications involved $2 \mathrm{~min}$ at $50{ }^{\circ} \mathrm{C}$ (stage 1 ), and $10 \mathrm{~min}$ at $95^{\circ} \mathrm{C}$ (stage 2 ), followed by 40 cycles of $95^{\circ} \mathrm{C}$ for $15 \mathrm{~s}$ and $60^{\circ} \mathrm{C}$ for $1 \mathrm{~min}$ (stage 3), and were carried out in 96-well optical PCR plates (N 801-0560, Perkin Elmer) placed in an automated fluorometer (ABI PRISM 7700 Sequence Detection System, Applied 
Biosystems). The different mRNA species were normalized against GAPDH determined in the same samples. The values obtained for resistant cells were subsequently divided with the values for parental cells to allow comparisons. Further details are available in Perkin-Elmer Instruction Manual of 1997.

\subsection{Array-based Comparative Genomic Hybridization (array-CGH)}

Genomic DNA was extracted from cultured cells using a commercial kit (Gen Elute, Sigma) and used for analyses of gene copy number alterations by array-CGH. The experimental procedures and data analyses were according to previously published information [14] . The tiling 33k or 38k BAC arrays used were produced at the SCIBLU Genomics Centre at Lund University, Sweden (www.lu.se/sciblu). Microarray slides were hybridized for $72 \mathrm{~h}$ at $37^{\circ} \mathrm{C}$ with differentially labeled test and reference DNA (Cy5-dCTP and Cy3-dCTP) in the presence of Cot-1 DNA, washed under stringent conditions, and nitrogen blow-dried.

Arrays were subsequently scanned using a GenePix 4200A (Axon instruments Inc., Union City, CA), and images were quantified with GenePix Pro 6.0 (Axon instruments, Wheatherford TX, USA) and uploaded in BioArray Software Environment, BASE (http://www.base.thep.lu.se/) [15] . The pin-based LOWESS algorithm [16] was used for data normalization. Relative copy numbers were determined from normalized $\log _{2}$ ratios, and altered regions were defined by CGH plotter [17] applying the thresholds $>0.25$ for gain, $>1.0$ for amplification, $<-0.25$ for loss and $<-1.0$ for homozygous loss. Mapping information and cytogenetic localization of clones were according to the UCSC genome browser (http:/www.genome.ucsc.edu/; July 2004 freeze). 


\section{Results}

As shown in Figure 2, as high as $30-40 \mu \mathrm{M}$ concentrations of 7-OHMTX, the primary catabolite of MTX, are readily attainable in the serum of childhood ALL patients treated with high-dose MTX [12] . Furthermore, while the parental drug MTX is efficiently cleared from the serum, relatively high levels (several $\mu \mathrm{M}$ ) of the catabolite 7-OHMTX that exceed those of the parent drug by $\sim 10$-fold, persist for a long time in the serum. Accordingly, we explored the pattern of gene expression by leukemic cells that had acquired resistance to MTX or 7-OHMTX. Wild type MOLT-4 cells and their sublines selected for resistance to MTX or 7-OHMTX were characterized regarding gene expression profiles (Fig. 3; Table 1) and DNA copy number alterations (Table 2). Gene expression levels were compared to gene copy numbers (Table 3) and verified for selected genes by qRT-PCR (Table 4).

\subsection{Expression Profiles of 7-OHMTX and MTX-Resistant cells}

Nine separate hybridizations were performed on the Human Genome U133A GeneChip representing triplicate inoculates of parental, 7-OHMTX- as well as MTX-resistant MOLT-4 cells. In total, 863 probe sets showed 2-fold or more difference in expression levels between the three lines analysed (Fig. 3). Analysis of the gene expression patterns revealed 176 genes in MTX-resistant cells and 365 genes in 7-OHMTX-resistant cells, the expression of which was more than 2-fold different from that in parental cells $(p<0.05)$ (Fig. 3). Forty-eight of these genes were common to both drug-resistant cell lines. Functional classification revealed frequent dysregulation of genes encoding signalling proteins in both resistant derivatives studied (Table 1). In the MTX-resistant subline, the expression of genes encoding proteins 
involved in DNA/RNA metabolism and membrane transport proteins were altered more profoundly, whereas in the 7-OHMTX-resistant cells, the most pronounced alterations were in transcripts coding for proteins involved in metabolism and cell proliferation (Table 1). The expression levels of certain genes with functions related to folate metabolism, purine biosynthetic pathway and transport of related substances are detailed in Table 3 . Both resistant cell lines exhibited 4-5-fold up-regulation of $M T H F R$. In addition, pronounced upregulation of more than 2-fold of MTR was detected in MTX-resistant cells, while 7OHMTX cells overexpressed FPGH, SLC19A2, PFAS, and GCHFR. Prominent underexpression involved APRT in MTX-resistant cells, whereas in 7-OHMTX-resistant cells, $A D A$ and $C B S$ were expressed at very low or undetectable levels.

\subsection{DNA Copy Number Alterations by Array-CGH}

To characterize alterations in gene copy numbers in parental and antifolate-resistant cells, each of these cell lines were first compared to normal reference DNA using array-CGH. Homozygous deletions common to all three cells were recorded at several chromosomal locations including 7q34, 9p21.2-p21.3, 11p14.3-15.1 and 14q11.2, while gene amplifications were not found. The target interval in 14q11.2, spans the loci for TRAV20 (gene segment for T-cell receptor alpha variable 20) and TRA genes. The region 9p21.3 encompasses the $C D K N 2 A$ gene locus encoding p16 and p14. In addition, regular gains or losses involved almost all chromosomes and were in most situations present in parental as well as drug-resistant cells (Table 2). Alterations present in drug-resistant cells are of interest since they may be related to the mechanism by which drug resistance has emerged. MTX-resistant cells were distinguished from parental cells by chromosomal 
gains within 5p, 6p, 7p, 7q, 8p, 12q, 17p, 17q, 18q and 22q as well as by loss in 10p. Cells resistant to 7-OHMTX differed from parental cells by gains involving $5 \mathrm{p}, 12 \mathrm{q}, 17 \mathrm{p}$ and loss of $1 \mathrm{p}, 10 \mathrm{p}$, and 13q. To further delineate acquired alterations, profiles were compared visually between parental and drug-resistant cells as a complementary approach to the scoring of copy number alterations by CGH plotter plugin. This revealed that several of the distinguishing alterations in resistant cells were present as close to borderline alterations at the threshold in parental cells. In addition, array-CGH experiments were carried out whereby resistant cells were hybridized against parental cells. This approach identified gene copy number alterations outside the $+/-0.25$ threshold relative to parental cells including increase in 8pter-q24.3 in MTX-resistant cells, as well as increases in1q21.1-qter and 12q23.2-qter and decreases in 10p11.22-p11.23 and 13q22.1-qter in 7-OHMTX cells.

\subsection{Corroboration of Array Expression Levels by qRT-PCR}

Analysis of the gene expression patterns of parental and resistant cells employing the Affymetrix HG-U133A microarray revealed consistent differences with respect to 9 genes, some of which encode proteins involved in folate metabolism or purine biosynthesis. These differences were confirmed by qRT-PCR (Table 4). The levels were normalized against the endogenous housekeeping gene $G A P D H$, which showed comparable expression levels in parental and antifolate-resistant cells. These analyses revealed similar levels of expression determined by microarray as well as by qRT-PCR for all nine genes analysed. The results also confirmed the extensive up-regulation of $M T R$ and $M T H F R$ along with down-regulation 
of $R F C$ in MTX-resistant cells, as well as up-regulation of $F P G H / G G H$ and $M T H F R$ in 7OHMTX-resistant cells (Table 4). 


\section{Discussion}

Concentrations of 7-OHMTX, the primary catabolite of MTX, as high as $30-40 \mu \mathrm{M}$, are readily attainable in the plasma of childhood ALL patients treated with high-dose MTX (Fig. 2) [12] . Furthermore, although MTX itself is efficiently cleared from the plasma, relatively high levels of 7-OHMTX (i.e. several $\mu \mathrm{M}$ ), that exceed those of the parent compound MTX by approximately 10 -fold, persist in the plasma for a long time. Accordingly, we herein explored the pattern of gene expression and gene dose alterations in human leukemia cells with acquired resistance to MTX or 7-OHMTX [11] . Copy number alterations were detected in parental cells as well as in both antifolate-resistant cell lines (Table 2). The genomic regions of acquired gains and losses do not include the genes whose products are known to be significant in response to MTX (Table 3). These findings suggest that drug-induced changes in gene expression do not stem from copy number alterations, but may instead be due to transcriptional regulatory alterations. Furthermore, gene amplifications were not detected, thereby demonstrating that increases in copy numbers of drug-resistance mediating genes were neither induced by treatment with MTX nor with exposure to 7-OHMTX. Expression of the RFC/SLC19A1 gene was down-regulated more than 2-fold in the MTXresistant cells, thereby confirming the role of this influx transporter as an important determinant of antifolate resistance [18-21] . However, the major finding of the present investigation is that the dramatic loss of FPGS activity in the 7-OHMTX cells, presumably associated with decreased intracellular levels of folate, was accompanied by consistent alterations in the expression of genes capable of preserving intracellular pools of reduced folate and/or increase purine nucleotide biosynthesis. Thus, among the major differences observed between these cell lines were the following (Fig. 4): (a) Expression of $A D A$ 
encoding the major enzyme of purine catabolism was down-regulated 10-fold in 7-OHMTX cells but unchanged in MTX cells. (b) Consistently, the cystathionine $\beta$ synthase $(C B S)$ gene was not expressed at all in 7-OHMTX cells, while being expressed 2-fold lower than the normal levels present in MTX-resistant cells. (c) Expression of genes encoding for MTHFR and GART, enzymes involved in methyl-tetrahydrofolate and purine biosyntheses, respectively, were elevated 5- and 2-fold in 7-OHMTX-resistant cells and 4- and 1.5-fold in MTX-resistant cells. (d) Furthermore, the levels of mRNAs encoding for the ATP-driven folate efflux transporters $M R P 1 / A B C C 1$ and $B C R P / A B C G 2$ were decreased in gene expression by 30\% and approximately 3-fold, respectively, in 7-OHMTX-resistant cells, whereas in MTX-resistant cells these levels were actually elevated 1.5- and 1.7-fold, respectively. These results are in accord with our recent findings that folate deprivation leads to a dramatic loss of $B C R P / A B C G 2$ expression as well as retention of this folate influx transporter in the cytoplasm rather than sorting it to the plasma membrane $[22,23]$. Furthermore, these findings are consistent with those reported recently by Evans and colleagues [24] who documented differential patterns of gene expression in various subtypes of bone marrow cells from newly diagnosed patients with B- or T-ALL. These investigators found that $42-44 \mathrm{hr}$ after MTX administration $\left(0.8 \mathrm{~g} / \mathrm{m}^{2}\right)$, T-ALL bone marrow cells formed long-chain (Glu4-7) MTX polyglutamates at a rate of only $355 \mathrm{pmol} / 10^{9}$ cells, while the corresponding rate for hyperdiploid B-cell leukemia was as high as $3,170 \mathrm{pmol} / 10^{9}$ cells. This difference was consistently associated with very low levels of FPGS mRNA in the TALL cells, as much as two orders of magnitude lower than the level present in B-lineage hyperdiploid cells, together with low level expression of the $B C R P / A B C G 2$ gene in T-ALL cells. In contrast, the levels of expression of the AICART and GARFT genes, encoding key 
enzymes involved in de novo purine biosynthesis were higher in the case of T-ALL than any other leukemia subtypes.

In agreement with the pronounced reduction observed in MTX uptake into MTX-resistant cells, diminished $R F C$ mRNA expression was observed by microarray (2-fold) and qRT-PCT (5-fold) in MTX-resistant leukaemia cells as compared to their parental counterpart (Table 4). However, the expression of $R F C$ was not altered in 7-OHMTX-resistant cells. Moreover, the level of FPGH mRNA was elevated more than 2-fold in 7-OHMTX-resistant cells. In contrast, no difference in FPGS expression was detected in either of the resistant cell lines suggesting that other mechanisms, e. g., post-transcriptional modifications, may be responsible for the low activity of this enzyme in these cells. In a recent study it has shown (M. Stark, C. Wichman, I. Avivi and Y.G. Assaraf; "Aberrant splicing of folylypolyglutamate synthetase as a novel mechanism of antifolate resistance in leukemia", Blood 2009, in press) that FPGS transcripts in antifolate-resistant ALL cell lines were highly aberrant as they underwent impaired splicing including exon skipping as well as intron retention. Hence, these novel alterations result in premature translation termination of FPGS, thereby leading to lack of FPGS activity and resistance to polyglutamatble antifolates. This mechanism was also corroborated in specimens from patients harbouring ALL, both at diagnosis and relapse. These findings provide a novel mechanistic basis for the loss of FPGS function in the absence of quantitative changes (i.e. decrease) in FPGS levels. Members of the family of multidrug resistance proteins, notably MRP1-5/ABCC15, and BCRP/ABCG2 are known to export the folate antagonist MTX out of cells [25-29]. Levels of $B C R P$ and MRP1-5 mRNAs were all slightly elevated (about $50 \%$ ) in the MTX-resistant subline, but unchanged in 7-OHMTX cells (Table 3). 
Cells resistant to 7-OHMTX displayed a dramatic up-regulation of VAMP8 (>700fold) ; VAMP8 is a vesicle-associated membrane protein involved in exocytosis, and it is hence tempting to speculate here that elimination of vesicle-sequestered 7OHMTX could be facilitated by VAMP 8 overexpression. This finding warrants further studies to explore this interesting possibility.

Among the genes that encode proteins involved in the folate pathway, the expression of MTHFR was most profoundly altered in both of our antifolate-resistant cell lines ( $>4$-fold upregulation) (Fig. 4). MTHFR plays a key role in folate metabolism by channelling singlecarbon units between nucleotide biosynthesis and methylation reactions. This enzyme converts 5,10-methylene-THF into 5-methyl-THF, the predominant circulating folate form that provides a methyl group for methylation of homocysteine to methionine. Moreover, 5,10 methylene-THF is required for TS activity and thymidylate biosythesis and its depletion could lead to dUTP misincorporation into DNA and a consequent increase in the frequency of chromosome damage, thereby facilitating the genotoxic effects of MTX. It has been reported that a deactivating MTHFR allele (the MTHFR C677T variant allele) can enhance the risk for ALL relapse potentially by increasing cellular levels of 5,10 Methylene-THF and deoxythymidine monophosphate (dTMP), thereby enhancing DNA synthesis and counteracting MTX cytotoxicity. Thus, the significant increment in the expression of MTHFR mRNA in both of our antifolate-resistant cell lines may be related to cellular tolerance to MTX and 7-OHMTX. Although diminished transport and lack of polyglutamylation can readily explain the mechanisms underlying the significant increase in resistance to MTX and 7-OHMTX, respectively, some alterations in gene expression of resistant cells may have contributed to their tolerance. 
In summary, the present characterization of the different patterns of gene expression profiles of MTX- and 7-OHMTX-resistant sublines of parental MOLT-4 leukemia cells selected by exposure to MTX and 7-OHMTX, provides a new perspective on differences in the pharmacodynamic consequences of HDMTX therapy. 7-OHMTX, the major metabolite of MTX exerts its cytotoxic effect by mechanisms that are distinct from those underlying the effects of the parent drug. Our current findings may facilitate the identification of the specific targets for MTX therapy and may provide additional insight into cellular responses to 7OHMTX. The distinct patterns of gene expression profiles in cells that have acquired resistance to MTX or 7-OHMTX may indicate that part of the gene expression profile observed following exposure to high doses of MTX reported earlier [30] is related to exposure to 7-OHMTX, rather than to MTX itself. The differential expression of a relatively large number of genes by 7-OHMTX-and MTX-resistant cells indicates the occurrence of separate and independent effects. Thus, in relation with HDMTX therapy, where higher concentrations of 7-OHMTX can be detected in the plasma, the pattern of gene expression will be different from that when low concentrations of 7-OHMTX are present. 


\section{References}

[1] Assaraf YG. Molecular basis of antifolate resistance. Cancer Metastasis Rev 2007;26:153-81.

[2] Fotoohi AK, Albertioni F. Mechanisms of antifolate resistance and methotrexate efficacy in leukemia cells. Leuk Lymphoma 2008;49:410-26.

[3] Gorlick R, Goker E, Trippett T, Waltham M, Banerjee D, Bertino JR. Intrinsic and acquired resistance to methotrexate in acute leukemia. N Engl J Med 1996;335:1041-8.

[4] Shafran A, Ifergan I, Bram E, Jansen G, Kathmann I, Peters GJ, et al. ABCG2 harboring the Gly482 mutation confers high-level resistance to various hydrophilic antifolates. Cancer Res 2005;65:8414-22.

[5] Volk EL, Schneider E. Wild-type breast cancer resistance protein (BCRP/ABCG2) is a methotrexate polyglutamate transporter. Cancer Res 2003;63:5538-43.

[6] Gifford AJ, Kavallaris M, Madafiglio J, Matherly LH, Stewart BW, Haber M, et al. P-glycoprotein-mediated methotrexate resistance in CCRF-CEM sublines deficient in methotrexate accumulation due to a point mutation in the reduced folate carrier gene. Int J Cancer 1998;78:176-81.

[7] Newton PA, Blakley RL. 7-Hydroxymethotrexate formation in a human lymphoblastic cell line. Biochem Biophys Res Commun 1984;122:1212-7.

[8] Farquhar D, Loo TL. Synthesis and biologic evaluation of 7hydroxymethotrexate, 7-methylaminopterin, and 7-methylmethotrexate. J Med Chem 1972;15:567-9.

[9] Drake JC, Allegra CJ, Baram J, Kaufman BT, Chabner BA. Effects on dihydrofolate reductase of methotrexate metabolites and intracellular folates formed following methotrexate exposure of human breast cancer cells. Biochem Pharmacol 1987;36:2416-8.

[10] Sholar PW, Baram J, Seither R, Allegra CJ. Inhibition of folate-dependent enzymes by 7-OH-methotrexate. Biochem Pharmacol 1988;37:3531-4.

[11] Fotoohi K, Jansen G, Assaraf YG, Rothem L, Stark M, Kathmann I, et al. Disparate mechanisms of antifolate resistance provoked by methotrexate and its metabolite 7-hydroxymethotrexate in leukemia cells: implications for efficacy of methotrexate therapy. Blood 2004;104:4194-201.

[12] Rask C, Albertioni F, Bentzen SM, Schroeder H, Peterson C. Clinical and pharmacokinetic risk factors for high-dose methotrexate-induced toxicity in children with acute lymphoblastic leukemia--a logistic regression analysis. Acta Oncol 1998;37:277-84.

[13] Albertioni F, Pettersson B, Beck O, Rask C, Seideman P, Peterson C. Simultaneous quantitation of methotrexate and its two main metabolites in biological fluids by a novel solid-phase extraction procedure using highperformance liquid chromatography. J Chromatogr B Biomed Appl 1995;665:163-70. 
[14] Jonsson G, Staaf J, Olsson E, Heidenblad M, Vallon-Christersson J, Osoegawa K, et al. High-resolution genomic profiles of breast cancer cell lines assessed by tiling BAC array comparative genomic hybridization. Genes Chromosomes Cancer 2007;46:543-58.

[15] Saal LH, Troein C, Vallon-Christersson J, Gruvberger S, Borg A, Peterson C. BioArray Software Environment (BASE): a platform for comprehensive management and analysis of microarray data. Genome Biol 2002;3:SOFTWARE0003.

[16] Yang MC, Ruan QG, Yang JJ, Eckenrode S, Wu S, McIndoe RA, et al. A statistical method for flagging weak spots improves normalization and ratio estimates in microarrays. Physiol Genomics 2001;7:45-53.

[17] Autio R, Hautaniemi S, Kauraniemi P, Yli-Harja O, Astola J, Wolf M, et al. CGH-Plotter: MATLAB toolbox for CGH-data analysis. Bioinformatics 2003;19:1714-5.

[18] Jansen G, Mauritz R, Drori S, Sprecher H, Kathmann I, Bunni M, et al. A structurally altered human reduced folate carrier with increased folic acid transport mediates a novel mechanism of antifolate resistance. J Biol Chem 1998;273:30189-98.

[19] Rothem L, Aronheim A, Assaraf YG. Alterations in the expression of transcription factors and the reduced folate carrier as a novel mechanism of antifolate resistance in human leukemia cells. J Biol Chem 2003;278:8935-41.

[20] Rothem L, Ifergan I, Kaufman Y, Priest DG, Jansen G, Assaraf YG. Resistance to multiple novel antifolates is mediated via defective drug transport resulting from clustered mutations in the reduced folate carrier gene in human leukaemia cell lines. Biochem J 2002;367:741-50.

[21] Rothem L, Stark M, Kaufman Y, Mayo L, Assaraf YG. Reduced folate carrier gene silencing in multiple antifolate-resistant tumor cell lines is due to a simultaneous loss of function of multiple transcription factors but not promoter methylation. J Biol Chem 2004;279:374-84.

[22] Ifergan I, Jansen G, Assaraf YG. Cytoplasmic confinement of breast cancer resistance protein (BCRP/ABCG2) as a novel mechanism of adaptation to shortterm folate deprivation. Mol Pharmacol 2005;67:1349-59.

[23] Ifergan I, Shafran A, Jansen G, Hooijberg JH, Scheffer GL, Assaraf YG. Folate deprivation results in the loss of breast cancer resistance protein (BCRP/ABCG2) expression. A role for BCRP in cellular folate homeostasis. J Biol Chem 2004;279:25527-34.

[24] Kager L, Cheok M, Yang W, Zaza G, Cheng Q, Panetta JC, et al. Folate pathway gene expression differs in subtypes of acute lymphoblastic leukemia and influences methotrexate pharmacodynamics. J Clin Invest 2005;115:110-7.

[25] Assaraf YG. The role of multidrug resistance efflux transporters in antifolate resistance and folate homeostasis. Drug Resist Updat 2006;9:227-46.

[26] Chen ZS, Lee K, Walther S, Raftogianis RB, Kuwano M, Zeng H, et al. Analysis of methotrexate and folate transport by multidrug resistance protein 4 (ABCC4): MRP4 is a component of the methotrexate efflux system. Cancer Res 2002;62:3144-50.

[27] Hooijberg JH, Broxterman HJ, Kool M, Assaraf YG, Peters GJ, Noordhuis P, et 
al. Antifolate resistance mediated by the multidrug resistance proteins MRP1 and MRP2. Cancer Res 1999;59:2532-5.

[28] Wielinga P, Hooijberg JH, Gunnarsdottir S, Kathmann I, Reid G, Zelcer N, et al. The human multidrug resistance protein MRP5 transports folates and can mediate cellular resistance against antifolates. Cancer Res 2005;65:4425-30.

[29] Zeng H, Bain LJ, Belinsky MG, Kruh GD. Expression of multidrug resistance protein-3 (multispecific organic anion transporter-D) in human embryonic kidney 293 cells confers resistance to anticancer agents. Cancer Res 1999;59:5964-7.

[30] Cheok MH, Yang W, Pui CH, Downing JR, Cheng C, Naeve CW, et al. Treatment-specific changes in gene expression discriminate in vivo drug response in human leukemia cells. Nat Genet 2003;34:85-90.

\section{Figure Legends}

Figure 1. The chemical structures of methotrexate (MTX) and its major catabolite 7hydroxymethotrexate (7-OHMTX).

Figure 2. Comparison of plasma levels for MTX and 7-OHMTX measured by HPLC in childhood ALL patients 24-90 hr after administration of high dose MTX as part of their treatment [12] . The lines for MTX and 7-OHMTX (bold) represent the median values for all 49 cases studied, and dotted and dashed lines indicate the +2SD of plasma levels of MTX and 7-OHMTX, respectively.

Figure 3. A, Gene expression patterns and $\mathbf{B}$, hierarchical clustering of parental cells (MOLT4/WT) and derivatives resistant to the MTX (MOLT4/MTX) or 7-OHMTX (MOLT4/7-OHMTX). Analyses based on three independent experiments were performed in GeneSpring 7.2, and the genes presented $(n=863)$ showed a more than 2-fold difference in expression level. $P<0.05$. 
Figure 4. Schematic illustration of dysregulated genes mediating the effects of MTX, as well as their relation to folate pathway and to purine, pyrimidine and methionine biosynthesis (modified from Fotoohi and Albertioni 2008) [2] .

Abbreviations are as follows: dihydrofolate reductase (DHFR); dihydrofolate (DHF); tetrahydrofolate (THF); S-adenosylmethionine (SAM); thymidylate synthetase (TS); deoxyuridine monophosphate (dUMP); deoxythymidine monophosphate (dTMP); Sadenosylhomocysteine (SAH); methylenetetrahydrofolate dehydrogenase 1 (MTHFD 1); 5,10-methylenetetrahydrofolate reductase (MTHFR); phosphoribosylglycinamide formyltransferase (GARFT); 5,10-methenyltetrahydrofolate synthetase (MTHFS), 5methyltetrahydrofolate-homocysteine methyltransferase (MTR); 5methyltetrahydrofolate-homocysteine methyltransferase reductase (MTRR); 5aminoimidazole-4-carboxamide ribonucleotide formyltransferase (ATIC or AICART); serine hydroxymethyltransferase 1 (SHMT 1); phosphoribosyl pyrophosphate amidotransferase (PPAT); cystathionine $\beta$-synthase (CBS); 10 - formyltetrahydrofolate (10-CHO-THF); 5,10 methylen tetrahydrofolate $(5,10=\mathrm{CH}-\mathrm{THF}) ; 5$ formyltetrahydrofolate (5-CHO-THF); 5 methyl tetrahydrofolate (5-CH3-THF). 
Figure 1

Figure 2.

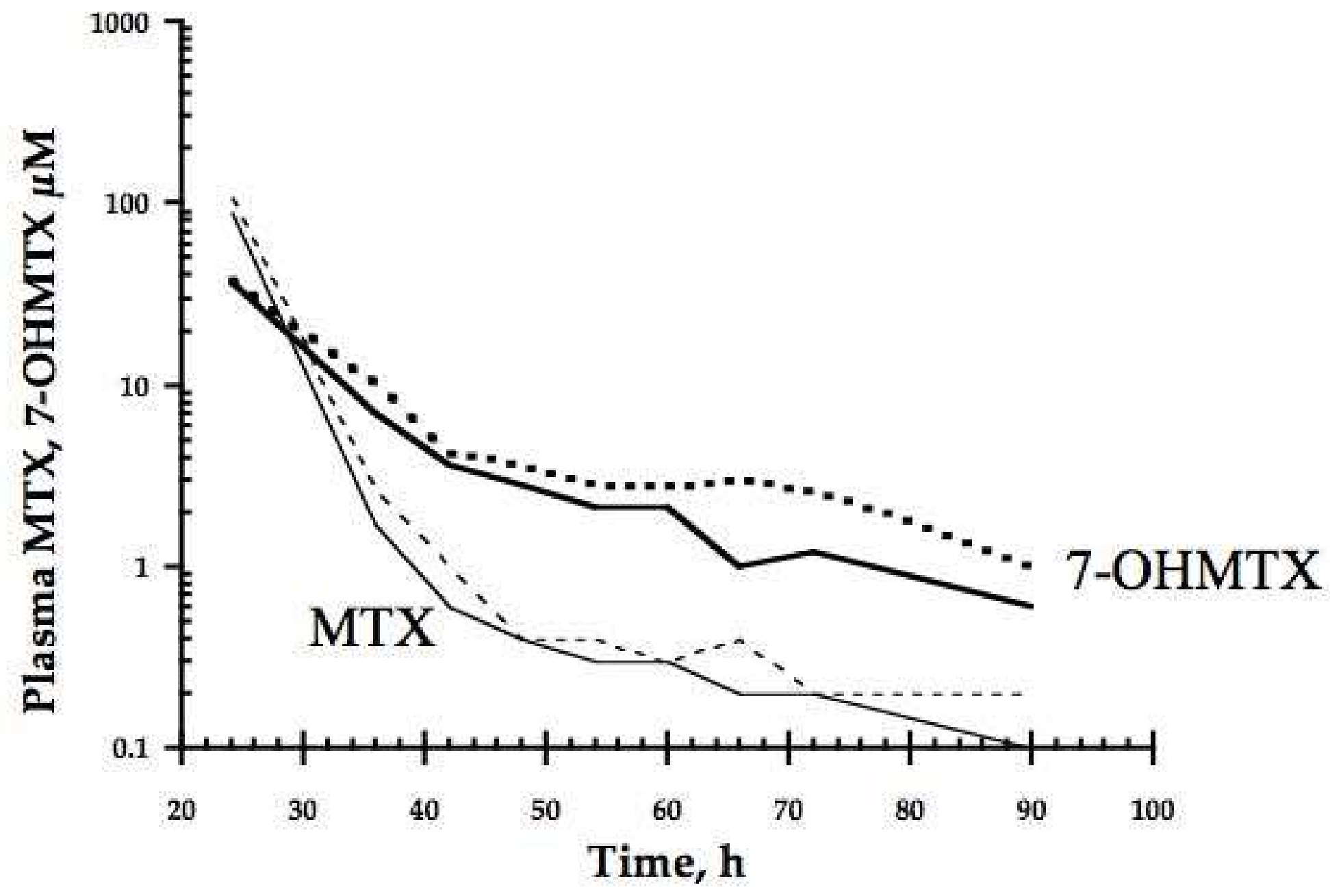


Figure 4

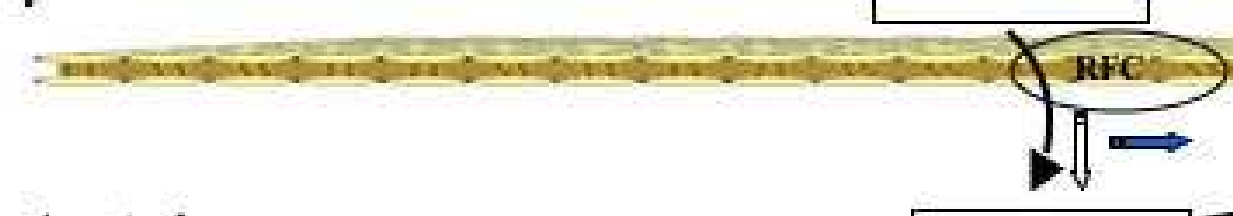

Apoptosis

k

Double stranded DNA breaks

Pyrimidine synthesis

R

DNA incorporetion
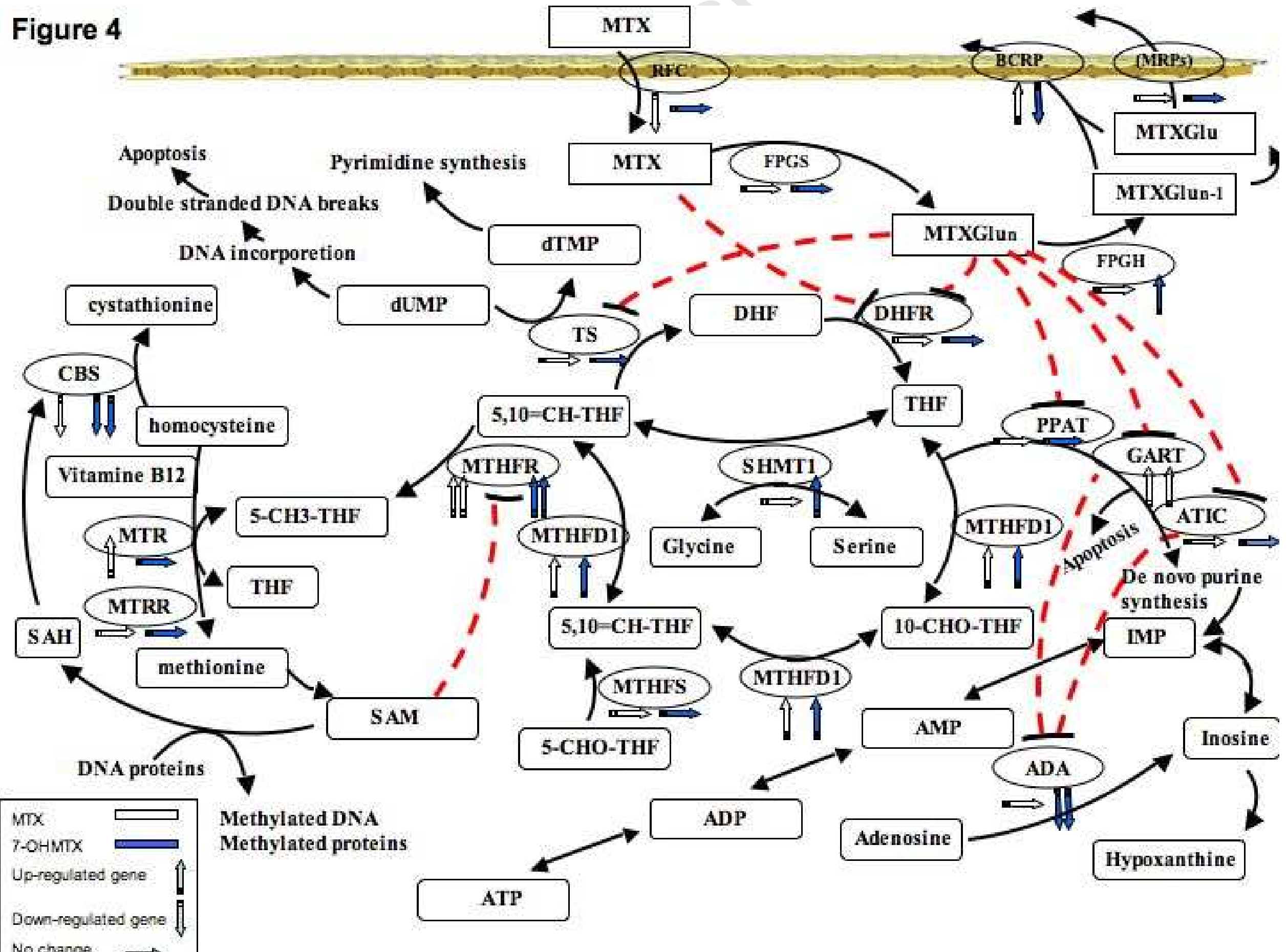

MTXGlun-1

rage $2 \zeta$ ot 30 
Table 1. Genes up- or down-regulated more than 2-fold in resistant versus parental cells.

\begin{tabular}{lccccc}
\hline & \multicolumn{2}{c}{ Up-regulated genes } & & \multicolumn{2}{c}{ Down-regulated genes } \\
\cline { 2 - 3 } \cline { 5 - 6 } Functional category & MTX & 7-OHMTX & & MTX & 7-OHMTX \\
\hline RNA / DNA metabolism & $10 \%(4)$ & $3 \%(4)$ & & $16 \%(22)$ & $10 \%(23)$ \\
Cell cycle & $0 \%(0)$ & $1 \%(1)$ & & $3 \%(4)$ & $1 \%(2)$ \\
Development & $5 \%(2)$ & $4 \%(5)$ & & $4 \%(6)$ & $10 \%(23)$ \\
Apoptosis & $5 \%(2)$ & $2 \%(3)$ & & $1 \%(1)$ & $4 \%(9)$ \\
Transporter & $10 \%(4)$ & $4 \%(5)$ & & $4 \%(6)$ & $5 \%(11)$ \\
Signaling & $30 \%(12)$ & $36 \%(49)$ & & $26 \%(35)$ & $33 \%(75)$ \\
Stress response & $5 \%(2)$ & $4 \%(5)$ & & $0 \%(0)$ & $1 \%(2)$ \\
Metabolism & $10 \%(4)$ & $22 \%(31)$ & & $17 \%(24)$ & $14 \%(32)$ \\
Growth & $15 \%(6)$ & $11 \%(15)$ & & $20 \%(27)$ & $15 \%(34)$ \\
Organization \& Proliferation & $0 \%(0)$ & $4 \%(5)$ & & $9 \%(13)$ & $7 \%(16)$ \\
Other & $10 \%(4)$ & $9 \%(13)$ & & $0 \%(0)$ & $0 \%(0)$ \\
Total & $100 \%(\mathrm{n}=40)$ & $100(\mathrm{n}=136)$ & $100(\mathrm{n}=138)$ & $100(\mathrm{n}=227)$ \\
\hline
\end{tabular}


Table 2. Summary of copy number aberrations by array-CGH in parental and resistant cells.

\begin{tabular}{|c|c|c|c|}
\hline \multicolumn{2}{|c|}{ Chr. Parental } & MTX resistant & 7-OHMTX resistant \\
\hline \multicolumn{4}{|c|}{ Copy number losses and homozygous deletions } \\
\hline 1 & 1q23.1-qter & 1q23.3-qter & $1 \mathrm{p} 36.31-\mathrm{p} 36.32$ \\
\hline 2 & $2 \mathrm{p} 24.1-\mathrm{p} 24.2$ & $2 \mathrm{p} 24.1-\mathrm{p} 24.2$ & $2 \mathrm{p} 24.1-\mathrm{p} 24.2$ \\
\hline 4 & $4 q 31.23$-qter & $4 \mathrm{q} 31.23$-qter & $4 q 31.23$-qter \\
\hline 5 & $5 q 13.2 ;$ q35.1-qter & $5 q 35.1-q$ ter & $5 q 13.2 ;$ q35.1-qter \\
\hline 7 & $7 p 21.3 \mathrm{hz} ; \mathrm{q} 34 \mathrm{hz}$ & $7 q 34 \mathrm{hz}$ & $7 p 21.3 h z ; q 34 h z$ \\
\hline 9 & 9p21.1-pter/p21.2-p21.3 hz/p23 hz & 9p21.1-pter/p21.2-p21.3hz/p23hz & 9p21.1-pter/p21.2-p21.3 hz \\
\hline 10 & - & 10p12.1-pter & 10p12.32-pter \\
\hline 11 & 11p14.3-p15.1hz; q25-qter & $11 p 14.3-p 15.1 h z ; q 25-q$ ter & $11 p 14.3-p 15.1 \mathrm{hz} ; \mathrm{q} 25$-qter \\
\hline 13 & - & - & $13 q 22.1-q 34$ \\
\hline 14 & $14 q 11.1-11.2 ; q 11.2 \mathrm{hz}$ & $14 q 11.2 \mathrm{hz}$ & $14 q 11.2 \mathrm{hz}$ \\
\hline 19 & $19 \mathrm{q} 13.2$ & $19 q 13.2$ & $19 q 13.2$ \\
\hline 22 & $22 \mathrm{q} 12.3$ & - & \\
\hline \multicolumn{4}{|c|}{ Copy number gains and amplifications } \\
\hline 1 & $1 \mathrm{p} 32.3 ; 1 \mathrm{p} 34.1-\mathrm{p} 36.31$ & $1 \mathrm{p} 32.2-36.31 ; 1 \mathrm{p} 36.3$ & $1 \mathrm{p} 32.3-\mathrm{p} 36.31$ \\
\hline 3 & $3 \mathrm{p} 21.1-\mathrm{p} 21.31$ & - & - \\
\hline 4 & 4 p16.3-pter & - & 4 p16.3-pter \\
\hline 5 & - & 5 p15.33-pter & 5p15.33-pter \\
\hline 6 & - & $6 \mathrm{p} 21.1-\mathrm{p} 22.1$ & - \\
\hline 7 & - & 7p22.1-pter; q11.22-q11.23 & - \\
\hline 8 & 8qter & 8p23.3-pter; p22-p21.3; qter & 8qter \\
\hline 9 & 9qter & 9qter & 9qter \\
\hline 10 & 10p11.22-p12.1; qter & 10p11.22-p12.1; qter & 10qter \\
\hline 12 & - & $12 \mathrm{q} 13.11-\mathrm{q} 14.1$ & $12 \mathrm{q} 21.33$-qter \\
\hline 14 & $14 q 32.2-q 32.33$ & $14 q 32.2-q 32.33$ & $14 q 32.2-q 32.33$ \\
\hline 16 & 16 p13.3-pter; q23.3-qter & 16 p13.3-pter; q23.3-qter & 16 p13.3-pter; q24.2-qter \\
\hline 17 & $17 q 25.1$-qter & $17 \mathrm{p} 13.1$-pter; q12-q21.33; q25.1-qter & 17p13.1-pter \\
\hline 18 & - & 18qter & - \\
\hline 19 & 19p12-pter; q13.31-q13.33 & 19p13.11-pter; q13.2; q13.31-qter & - \\
\hline 20 & 20pter-qter & 20pter-qter & 20pter-qter \\
\hline 21 & 21q22.3-qter & 21q22.3-qter & - \\
\hline 22 & $22 \mathrm{q} 13.1-\mathrm{q} 13.2 ;$ qter & $22 \mathrm{q} 11.1-\mathrm{q} 11.23 ; \mathrm{q} 12.3$-qter & 22qter \\
\hline
\end{tabular}

Homozygous losses hz are indicated in italic and bold; Chr. $=$ Chromosome 
Table 3. Expression levels of selected genes and copy number of cytoband involved.

\begin{tabular}{|c|c|c|c|c|c|c|c|}
\hline \multirow{2}{*}{$\begin{array}{l}\text { Gene } \\
\text { symbol }\end{array}$} & & \multicolumn{2}{|c|}{ Expression-array } & \multirow{2}{*}{$\begin{array}{l}\text { Cytoband } \\
\text { concerned }\end{array}$} & \multicolumn{3}{|c|}{ Array-CGH } \\
\hline & & MTX & 7-OHMTX & & Parental & MTX & 7-OHMTX \\
\hline$F P G S$ & folylpolyglutamate synthetase & 1.0 & 1.0 & $9 q 34.11$ & gain & gain & gain \\
\hline$F P G H / G G H$ & folylpolygammaglutamyl hydrolase, gamma-glutamyl hydrolase & 1.2 & 3.0 & $8 \mathrm{q} 12.3$ & n.a & n.a & n.a \\
\hline MTR & 5-methyltetrahydrofolate-homocysteine methyltransferase, & 2.8 & 1.3 & $1 \mathrm{q} 43$ & loss & loss & n.a \\
\hline$A I C A R T$ & 5-aminoimidazole-4-carboxamide ribonucleotide formyltransferase & 1.0 & 1.0 & $2 q 35$ & n.a & $\mathrm{n}-\mathrm{a}$ & na. \\
\hline$R F C / S L C 19 A 1$ & solute carrier family 19 (folate transporter) member 1 & 0.4 & 0.9 & $21 \mathrm{q} 22.3$ & gain & n.a & n.a \\
\hline$G A R T$ & phosphoribosylglycinamide formyltransferase & 1.6 & 1.8 & $21 \mathrm{q} 22.11$ & n.a & n.a & n.a \\
\hline MTHFR & 5,10-methylenetetrahydrofolate reductase (NADPH) & 4.3 & 4.8 & $1 \mathrm{p} 36.22$ & gain & gain & gain \\
\hline MRP4 & ATP-binding cassette, sub-family C (CFTR/MRP) member 4 & 1.4 & 0.9 & $13 q 32.1$ & n.a & n.a & loss \\
\hline MRP5 & ATP-binding cassette, sub-family C (CFTR/MRP) member 5 & 1.6 & 1.2 & $3 q 27.1$ & n.a & n.a & n.a \\
\hline MTHFD1 & methylenetetrahydrofolate dehydrogenase 1 & 1.4 & 1.5 & $14 \mathrm{q} 23.2$ & n.a & n.a & n.a \\
\hline MTHFD2 & methylenetetrahydrofolate dehydrogenase 2 & 1.2 & 1.8 & $2 \mathrm{p} 13.1$ & n.a & n.a & n.a \\
\hline DHFR & dihydrofolate reductase & 1.4 & 1.0 & $18 \mathrm{q} 11.2$ & n.a & n.a & n.a \\
\hline$T S / T Y M S$ & thymidylate synthetase & 0.6 & 0.7 & $18 \mathrm{p} 11.32$ & n.a & n.a & n.a \\
\hline MTRR & 5-methyltetrahydrofolate-homocysteine methyltransferase reductase & 1.1 & 0.9 & $5 \mathrm{p} 15.31$ & n.a & n.a & n.a \\
\hline MTHFS & 5,10-methenyltetrahydrofolate synthetase & 1.0 & 0.9 & $15 q 25.1$ & n.a & n.a & n.a \\
\hline GCH1 & GTP cyclohydrolase 1 (dopa-responsive dystonia) & 1.4 & 0.9 & $14 \mathrm{q} 22.2$ & n.a & n.a & n.a \\
\hline SLC19A2 & solute carrier family 19 (thiamine transporter) member 2 & 1.4 & 2.1 & $1 \mathrm{q} 24.2$ & loss & loss & n.a \\
\hline SHMT1 & serine hydroxymethyltransferase 1 & 1.1 & 2.0 & $17 \mathrm{p} 11.2$ & n.a & n.a & n.a \\
\hline$B C R P / A B C G 2$ & ATP-binding cassette, sub-family G (WHITE) member 2 & 1.7 & 0.4 & $4 \mathrm{q} 22.1$ & n.a & n.a & n.a \\
\hline$P F A S$ & phosphoribosylformylglycinamidine synthase (FGAR amidotransferase) & 1.9 & 2.5 & $17 \mathrm{p} 13.1$ & n.a & gain & n.a \\
\hline GCHFR & GTP cyclohydrolase I feedback regulator & 0.6 & 7.7 & $15 \mathrm{q} 15.1$ & n.a & n.a & n.a \\
\hline$I T P A$ & inosine triphosphatase (nucleoside triphosphate pyrophosphatase) & 0.6 & 0.9 & $20 \mathrm{p} 13$ & gain & gain & gain \\
\hline$d G K / D G U O K$ & deoxyguanosine kinase, dGK/ DGUOK & 1.0 & 1.8 & $2 \mathrm{p} 13.1$ & n.a & n.a & n.a \\
\hline$A P R T$ & adenine phosphoribosyltransferase & 0.4 & 0.6 & $16 \mathrm{q} 24.3$ & gain & gain & gain \\
\hline$A D A$ & adenosine deaminase & 0.9 & 0.1 & $20 \mathrm{q} 13.2$ & gain & gain & gain \\
\hline PPAT & phosphoribosyl pyrophosphate amidotransferase & 1.1 & 1.3 & $4 \mathrm{q} 12$ & n.a & n.a & n.a \\
\hline$M R P 1 / A B C C 1$ & ATP-binding cassette, sub-family C (CFTR/MRP) member 1 & 1.5 & 0.7 & $16 \mathrm{p} 13.11$ & n.a & n.a & n.a \\
\hline$M R P 2 / A B C C 2$ & ATP-binding cassette, sub-family C (CFTR/MRP) member 2 & 0.8 & 0.8 & $10 \mathrm{q} 24.2$ & n.a & n.a & n.a \\
\hline$M R P 3 / A B C C 3$ & ATP-binding cassette, sub-family C (CFTR/MRP) member 3 & 1.0 & 1.0 & $17 \mathrm{q} 21.33$ & n.a & gain & n.a \\
\hline P-glycoprotein & ATP-binding cassette, sub-family B (MDR/TAP) member 1 & 1.2 & 0.9 & $7 q 21.12$ & n.a & n.a & n.a \\
\hline$C B S$ & cystathionine $\beta$-synthase & 0.5 & $\mathbf{0 . 0}$ & $21 \mathrm{q} 22.3$ & gain & gain & n.a \\
\hline
\end{tabular}

Expression levels are in relation to parental cells (MTX / parental or 7-OHMTX / parental) giving an arbitrary value of 1.0 for parental cells

DNA copy number are in relation to normal reference DNA; $n . a=$ no aberration detected

Expression changes greater than 2-fold and copy number aberrations above thresholds are indicted in bold 
Table 4. Expression of selected genes by array and qRT-PCR in resistant as compared to parental cells. by Affymetrix array by qRT-PCR

\begin{tabular}{|c|c|c|c|c|c|c|}
\hline & & & & & \\
\hline & Parental & MTX & 7-OHMTX & Parental & MTX & 7-OHMTX \\
\hline$\overline{F P G S}$ & $(1.0)$ & 1.0 & 1.0 & $(1.0)$ & $0.83 \pm 0.02$ & $1.0 \pm 0.20$ \\
\hline$F P G H / G G H$ & $(1.0)$ & 1.2 & 3.0 & $(1.0)$ & $0.76 \pm 0.05$ & $2.0 \pm 0.38$ \\
\hline$M T R$ & $(1.0)$ & 2.8 & 1.3 & (1.0) & $1.60 \pm 0.24$ & $1.1 \pm 0.10$ \\
\hline ATIC & (1.0) & 1.0 & 1.0 & (1.0) & $0.87 \pm 0.03$ & $1.0 \pm 0.05$ \\
\hline$R F C / S L C 19 A 1$ & $(1.0)$ & 0.4 & 0.9 & $(1.0)$ & $0.20 \pm 0.03$ & $2.2 \pm 0.37$ \\
\hline$G A R T$ & $(1.0)$ & 1.6 & 1.8 & (1.0) & $1.76 \pm 0.25$ & $1.6 \pm 0.16$ \\
\hline MTHFR & $(1.0)$ & 4.3 & 4.8 & (1.0) & $2.80 \pm 0.40$ & $2.3 \pm 0.20$ \\
\hline$M R P 4$ & $(1.0)$ & 1.4 & 0.9 & (1.0) & $1.30 \pm 0.18$ & $1.2 \pm 0.08$ \\
\hline MRP5 & $(1.0)$ & 1.6 & 1.2 & $(1.0)$ & $1.40 \pm 0.11$ & $1.9 \pm 0.16$ \\
\hline
\end{tabular}

Expression values are in relation to parental cells (MTX / parental or 7-OHMTX / parental), giving an arbitrary value of 1.0 in parental cells as indicated 
Resistant to methotrexate and 7-hydroxymethotrexate reveals alterations that preserve intracellular levels of folate and nucleotide biosynthesis

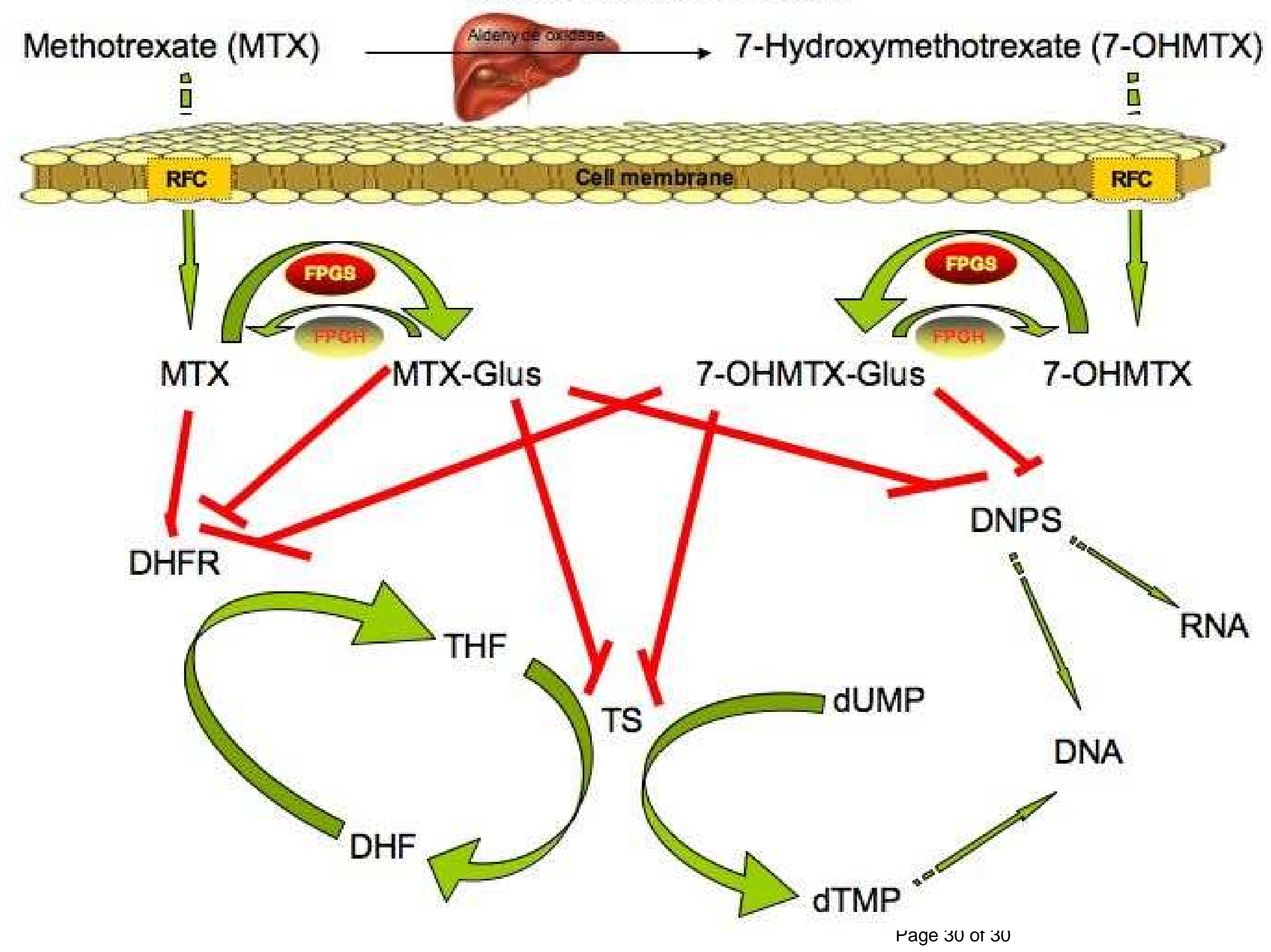



